\title{
THE
}

$11-15-1991$

\section{Deterministic and Stochastic Spin Diffusion in Classical Heisenberg Magnets}

Jian-Min Liu

University of Rhode Island

Niraj Srivastava

University of Rhode Island

V. S. Viswanath

University of Rhode Island

Gerhard Müller

University of Rhode Island, gmuller@uri.edu

Follow this and additional works at: https://digitalcommons.uri.edu/phys_facpubs

Terms of Use

All rights reserved under copyright.

\section{Citation/Publisher Attribution}

Jian-Min Liu, Niraj Srivastava, V.S. Viswanath and Gerhard Müller. Deterministic and stochastic spin diffusion in classical Heisenberg magnets. J. Appl. Phys. 70 (1991), 6181-6183.

Available at: http://dx.doi.org/10.1063/1.350037 


\title{
Deterministic and stochastic spin diffusion in classical Heisenberg magnets
}

\author{
Jian-Min Liu, Niraj Srivastava, V. S. Viswanath, and Gerhard Muller \\ Department of Physics, The University of Rhode Island, Kingston, Rhode Island 02881-0817 \\ This computer simulation study provides further evidence that spin diffusion in the one- \\ dimensional classical Heisenberg model at $T=\infty$ is anomalous: $\left\langle\mathbf{S}_{j}(t) \cdot \mathbf{S}_{j}\right\rangle \sim t^{-\alpha_{1}}$ with \\ $\alpha_{1}>1 / 2$. However, the exponential instability of the numerically integrated phase-space \\ trajectories transforms the deterministic transport of spin fluctuations into a \\ computationally generated stochastic process in which the global conservation laws are still \\ satisfied to high precision. This may cause a crossover in $\left\langle\mathbf{S}_{j}(t) \cdot \mathbf{S}_{j}\right\rangle$. from anomalous \\ spin diffusion $\left(\alpha_{1}>1 / 2\right)$ to normal spin diffusion $\left(\alpha_{1}=1 / 2\right)$ at some characteristic time lag \\ that depends on the precision of the numerical integration.
}

For magnetic insulators with isotropic exchange interaction between nearest-neighbor spins on a $d$-dimensional lattice, such as the Heisenberg model:

$$
\mathrm{H}=-J \sum_{\langle i j\rangle} \cdot \mathbf{S}_{i} \cdot \mathbf{S}_{j}
$$

the dominant transport mechanism of spin fluctuations is diffusion. Spin diffusion is a phenomenological concept widely employed in the analysis and interpretation of dynamical experiments (NMR, ${ }^{1} \mathrm{ESR}^{2}$ ) on insulating magnetic compounds.

Theoretically, it has turned out to be extremely difficult to establish, under what circumstances the rapid deterministic transverse spin motion; specified by the equations of motion

$$
\dot{\mathbf{S}}_{i}=-\mathbf{S}_{i} \times \partial H / \partial \mathbf{S}_{i}
$$

for individual classical 3-component spins $\mathbf{S}_{i}$, gives rise to slow stochastic longitudinal spin motion, specified by the diffusion equation

$$
\frac{\partial}{\partial t} \mathbf{S}(\mathbf{q}, t)=-D q^{2} \mathbf{S}(\mathbf{q}, t)
$$

for the spin variables ${ }^{3,4}$

$$
\mathbf{S}(\mathbf{q}, t)=\frac{1}{\sqrt{N}} \sum_{j} \exp \left(\mathrm{iq} \cdot \mathbf{r}_{j}\right) \mathbf{S}_{j}(t) .
$$

The validity of the phenomenological Eq. (3) implies that the correlation function $C(\mathbf{q}, t)=\langle\mathbf{S}(\mathbf{q}, t)$ $\cdot \mathbf{S}(-\mathbf{q}, 0)\rangle$ decays exponentially in time,

$$
C(\mathbf{q}, t) \sim e^{-D q^{2} t},
$$

for small $q$ and large $t$, and that the spin autocorrelation function $C_{0}(t)=\left\langle\mathbf{S}_{j}(t) \cdot \mathbf{S}_{j}(0)\right\rangle$ exhibits a distinctive power-law long-time tail,

$$
C_{0}(t) \sim t^{-\alpha_{d}}
$$

The characteristic exponent predicted by spin diffusion phenomenology has the value $\alpha_{d}^{(S D)}=d / 2$.

In a recent computer simulation study, ${ }^{5}$ the function $C_{0}(t)$ at $T=\infty$ for the classical Heisenberg model (1) in dimensionalities $d=1,2,3$ was analyzed in some detail, and the diffusive long-time tail was clearly identified for the first time. However, the characteristic exponent $\alpha_{d}$ inferred from the simulation data was found to deviate significantly from the value $\alpha_{d}^{(S D)}$. The largest deviation was found for the case $d=1$. This was interpreted as evidence for anomalous spin diffusion. But that conclusion was challenged by Gerling and Landau, ${ }^{6,7}$ whose more extensive simulation study of the $d=1$ case demonstrated that the slope $\bar{\alpha}_{1}$ of $\dot{C}_{0}(\dot{t})$ in a log-log plot has a trend of decreasing magnitude for increasing $t$. The extrapolation of that trend was interpreted to be consistent with normal spin diffusion: $\bar{\alpha}_{1} \rightarrow \alpha_{1}^{(S D)}=1 / 2$.

Our own conclusions drawn from the two simulation studies $^{5-7}$ may be summarized as follows: (i) The anomalous character of the diffusive long-time tail persists out to the largest value of $t$ for which a quantitative analysis of the slope yields reliable results. (ii) The slope $\bar{\alpha}_{1}$ inferred from the simulation data decreases from a value $\bar{\alpha}_{1} \simeq 0.60$ at small $t$ to a value $\bar{\alpha}_{1} \simeq 0.57-0.58$ at $J t=80$ (depending on the data used and the type of analysis employed). (iii) The question of whether the truly asymptotic behavior of the long-time tail is anomalous $\left(\alpha_{1}>1 / 2\right)$ or in agreement with spin diffusion phenomenology $\left(\alpha_{1}=1 / 2\right)$ has eluded a conclusive answer thus far. The present study yields additional evidence in support of the conclusion that anomalous spin diffusion is real, as we shall see.

How does the computational error in the numerical integration of the simulation affect the decay of the spin autocorrelation function? From a superficial point of view the matter seems simple and straightforward. If it wasn't for the conservation law $\mathbf{S}_{T}=$ const, the function $C_{0}(t)$ would decay exponentially in $t$ instead of algebraically. Hence a numerical error in the integration which causes a violation of the conservation law (albeit small) amounts to a partial removal of the constraint that prevents exponential decay. The effect to be expected is a more rapidly decaying function $C_{0}(t)$, reflected in the data analysis by a larger (more anomalous) value of $\bar{\alpha}_{1}$. In reality, the connection between cause and effect is more subtle, and may produce a more slowly decaying function $C_{0}(t)$.

The subtlety that allows for such a paradoxical result derives from the nature of the Liouvillian flow in the $2 N$ - 


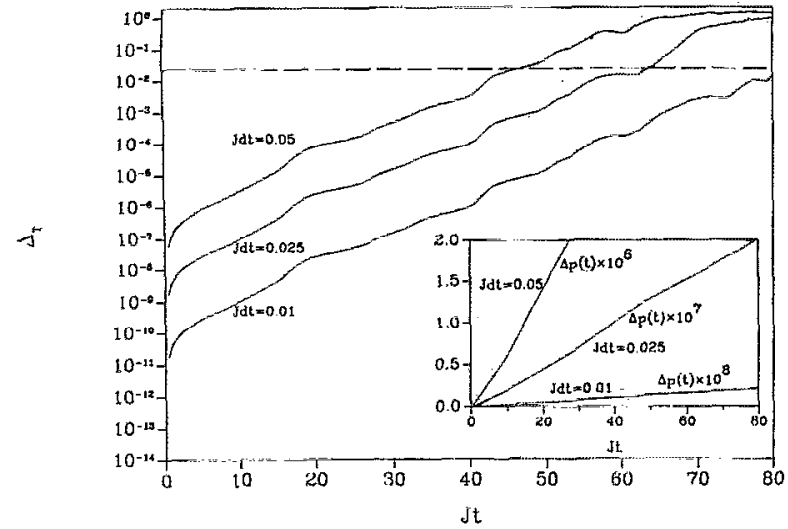

FIG. 1. Computational error $\Delta_{T}(t)$ tangential to global invariants (main plot) and computational error $\Delta_{p}(t)$ perpendicular to global invariants (inset) for a randomly chosen phase-space trajectory. The integration was performed by RK4 with fixed time step: $J d t=0.05,0.025,0.01$, respectively, for the three lines. For better visual effect, the curves in the inset have different vertical scales. The horizontal dashed line in the main plot indicates the value of the spin autocorrelation function $C_{0}(t)$ at $J t=80$.

dimensional phase space of the Heisenberg model with $N$ classical spins. That phase space is foliated by lower-dimensional invariant surfaces. Their dimensionality depends on the number of existing analytic invariants: the total energy $H$, the total spin $\mathbf{S}_{T}$, and perhaps some less obvious ones. In a nonintegrable many-body system the dimensionality of the invariant surfaces is believed to be not much lower than $2 N$. The motion of any individual phase point is confined to an invariant surface, but the flow of phase points on an invariant surface is chaotic.

The numerical integration of the equations of motion (2) is subject to two different types of computational error: $\square$ Deviations $\Delta_{T}(t)$ tangential to the invariant surface: The (chaotic) trajectories of two nearby phase points tend to separate exponentially in $t$. This implies an exponential error propagation in the numerical integration. It causes a loss of $M$ significant digits per time interval $M \Delta t_{0}^{(T)}$ on average. $\square$ Deviations $\Delta_{P}(t)$ perpendicular to the invariant surface: A computational trajectory also tends to stray off the invariant surface on which it starts out, but at a much slower rate, namely linearly in $t$. The associated loss of $M$ significant digits occurs on a time interval $10^{M-1} \Delta t_{0}^{(P)}$ on average.

For the purpose of illustration, we have determined the time-dependence of the following two quantities for a single phase-space trajectory:

$\Delta_{T}(t)=\hat{T}(t / 2)\left\{N^{-1} \sum_{i=1}^{N} \sum_{\alpha=x y z}\left[S_{i}^{\alpha}(t)-S_{i}^{\alpha}(0)\right]^{2}\right\}^{1 / 2}$,

$\Delta_{P}(t)=\widehat{T}(t / 2)|H(t)-H(0)|$,

where $\widehat{T}(t / 2)$ symbolizes the property that the spin motion is reversed at time $t / 2$ in the numerical integration. We have used a 4th-order Runge-Kutta method with fixed time step. The two quantities in Eqs. (6) and (7) are plotted in Fig. 1, the former with an exponential scale on the vertical axis (main plot) and the latter with a linear vertical scale (inset). The three curves in each plot represent data from integrations with different time step $d t$.

The results clearly demonstrate the exponential and linear error propagation in the two quantities $\Delta_{T}(t)$ and $\Delta_{P}(t)$, respectively. In both quantities, the rate of error propagation increases with increasing time step $d t$. The function $\Delta_{T}(t)$ is observed to level off when its size reaches $0(1)$. The value of $C_{0}(t)$ at $J t=80$ is about 0.023 (indicated by a dashed line in Fig. 1). The computational error $\Delta_{T}(t)$ is expected to become non-negligible when it has grown to a size comparable to $C_{0}(t)$. This is the case at $J t<80$ at least for the two larger time steps used. The function $\Delta_{P}(t)$, by contrast, stays orders of magnitude below the value of $C_{0}(t)$ at $J t=80$ for all three time steps used. The important point for the analysis of simulation data is that the two types of computational error, $\Delta_{T}(t)$ and $\Delta_{P}(t)$, have a quite different effect on the diffusive long-time tail of the spin autocorrelation function.

We have performed a simulation on systems with $N$ $=250$ spins and periodic boundary conditions, using RK4 integrations with time step $J d t=0.01$ and another simulation with the larger time step $J d t=0.05$. For both simulations, the function $C_{0}(t)$ has been computed from some 200000 independent integrations with randomly chosen initial conditions. It is customary to plot the long-time tail of $C_{0}(t)$ in a $\log -\log$ graph and determine the exponent $\alpha_{1}$ from the slope $\bar{\alpha}_{1}$ (see Refs. 5-7). Here we go one step further in the analysis before plotting the results: We determine the slope $\bar{\alpha}_{1}$ by linear regression from 151 consecutive data points $\left(\ln \left[C_{0}(t)\right], \ln (J t)\right)$ (amounting to an interval $J t_{\mathrm{av}}=30$ ) and plot the result versus $1 / J t$ (midpoint of interval). This representation is very useful and accurate for the determination of $\alpha_{1}$, but it requires a fairly low level of statistical fluctuations in the simulation data. In our case, the simulation data are sufficiently smooth up to $J t$ $\approx 60$, yielding data points in Fig. 2 up to $J t \simeq 45$. A somewhat similar plot of simulation data can be found in Fig. 2 of Ref. 7.

Consider first the results shown in the main plot of our Fig. 2, obtained from RK4 with $J d t=0.01$. The value of $\overline{\alpha_{1}}$ decreases monotonically, almost linearly with decreasing $1 / J t$, and extrapolates to an asymptotic value $\alpha_{1}$ between 0.56 and 0.57 . These simulation data represent the most unambiguous numerical evidence to date in support of the proposition that spin diffusion in the spin autocorrelation function of the classical $d=1$ Heisenberg model is anomalous.

Looking now at the results shown in the inset to Fig. 2, obtained from RK 4 with $d t=0.05$, we observe that a very similar trend persists for exactly as long as the computational error $\Delta_{T}$ has not yet caught up with the value of $C_{0}(t)$. That happens at $J t \simeq 45$ (see Fig. 1). The first interval of size $J t_{\mathrm{av}}=30$ which overlaps with this point has its midpoint at $J t \simeq 30$. This is the data point marked by an arrow in Fig. 2. Here the slope function $\bar{\alpha}_{1}(t)$ changes trend toward a smaller asymptotic value of the exponent $\alpha_{1}$.

There are two interrelated properties of these data which call for an explanation: (i) The value of the char- 


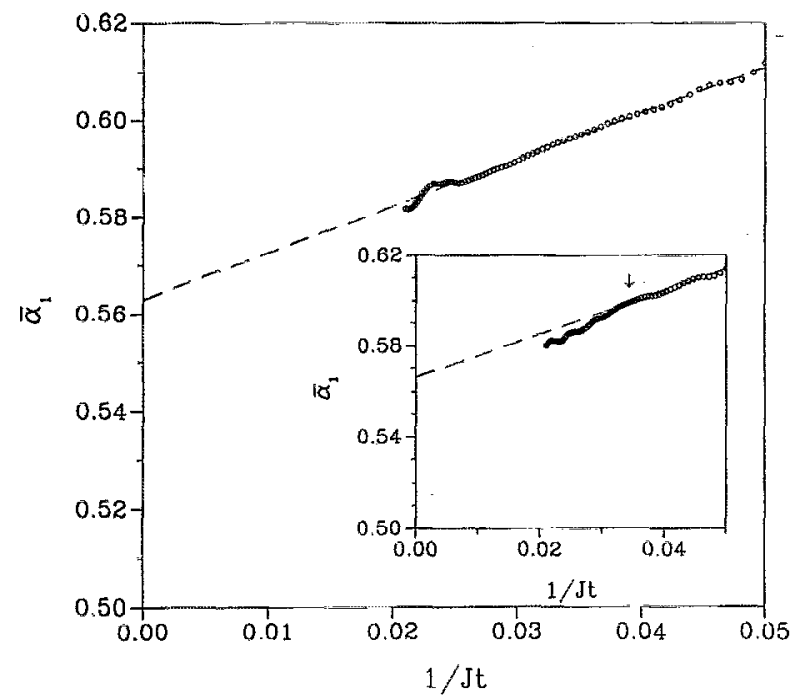

FIG. 2. Effective characteristic exponent $\bar{\alpha}_{1}$ as determined from the slope of the long-time tail of $C_{0}(t)$ in a $\log -\log$ plot. Each data point for $\bar{\alpha}_{1}$ is determined by linear regression from 151 consecutive data points $\left(\ln \left[C_{0}(t)\right], \ln (J t)\right)$ spaced at $J \Delta t=0.2$ and plotted versus $1 / J t$ at the midpoint of the total interval. Shown are results for two independent simulations. The number of random initial conditions used is 199500 (main plot) and 200000 (inset). Both simulations employ a 4th-order Runge-Kutta integration, one with fixed time step $J d t=0.01$ (main plot), the other with fixed time step $J d t=0.05$ (inset).

acteristic exponent $\alpha_{1}$ appears to be affected by the computational error $\Delta_{T}(t)$ associated with the exponential instability of numerically integrated phase-space trajectories. (ii) The simulation which uses higher precision in the numerical integration tends to yield a more rapidly decaying long-time tail in $C_{0}(t)$ than the one which uses lower precision. That is the paradox mentioned previously.

These properties are in contradiction to the assumptions underlying spin diffusion phenomenology. Note that at the point where the computational error $\Delta_{T}(t)$ has grown to a size comparable to that of $C_{0}(t)$, all global conservation laws including the one $\left(\mathbf{S}_{T}=\right.$ const) on which the spin diffusion phenomenon hinges are still satisfied to high precision. Moreover, the robustness of the spin diffusion phenomenology derives from the fact that it depends (in addition to the aforementioned conservation law) only on the mixing character of the phase flow and on certain rather weak requirements for the transport coefficient (diffusivity), but not on such detailed properties of the phase flow as might be affected by the computational error $\Delta_{T}(t)$.
The properties (i) and (ii) of our data, if they can be confirmed by more extensive and systematic simulation studies, suggest the following intriguing scenario: The fact is that the exponential instability of numerically integrated phase-space trajectories alters the nature of the microscopic dynamics from a nonlinear deterministic process to a computationally generated stochastic process. This is a consequence of the inevitable deterministic randomness, an intrinsic property of Hamiltonian chaos. Since both microscopic processes satisfy the same global conservation laws, both support a diffusive long-time tail (5) in the spin autocorrelation function. That function represents a contracted-level description for either of the two processes. On that level of description, the two are indistinguishable at least until the computational error $\Delta_{T}(t)$ has grown to significant size. When this happens, such as near the arrow in Fig. 2, we may see the crossover between two different spin diffusion processes, which we call deterministic spin diffusion and stochastic spin diffusion, respectively, in reference to the nature of the underlying microscopic dynamics. While the manifestly anomalous long-time tail of the deterministic process implies a singular transport coefficient, it may well be that its stochastic counterpart produces a well-behaved transport coefficient and thus gives rise to normal spin diffusion.

This scenario, if it is valid, resolves the paradox quite elegantly. However, it does still not solve the puzzle which provoked the paradox: an understanding of anomalous spin diffusion. More theoretical and computational efforts are clearly needed.

This work was supported by the U.S. National Science Foundation, Grant No. DMR-90-07540. Access to supercomputers at the National Center for Supercomputing Applications, University of Illinois at Urbana-Champaign and at NASA-Ames Research Center are gratefully acknowledged.

${ }^{1}$ D. Hone, C. Scherer, and F. Borsa, Phys. Rev. B 9, 965 (1974); F. Borsa and M. Mali, Phys. Rev. B 9, 2215 (1974); J-P. Boucher et al. Phys. Rev. B 13, 4098 (1976).

${ }^{2}$ H. Benner, Phys. Rev. B 18, 319 (1978); A. Lagendijk and E. Siegel, Solid State Commun. 29, 709 (1976).

${ }^{3}$ D. Forster, Hydrodynamic Fluctuations, Broken Symmetry and Correlation Functions (Benjamin, Reading, 1975).

${ }^{4}$ H. C. Fogedby and A. P. Young, J. Phys. C 11, 527 (1978).

${ }^{5}$ G. Müller, Phys. Rev. Lett. 60, 2787 (1988); 63, 813 (1989).

${ }^{6}$ R. W. Gerling and D. P. Landau, Phys. Rev. Lett. 63, 812 (1989).

${ }^{7}$ R. W. Gerling and D. P. Landau, Phys. Rev. B 42, 8214 (1990). 